\title{
Weitere Untersuchungen zur Morphologie, Verbreitung und Ökologie von Stephanoscyphus planulophorus (Scyphozoa, Coronata)
}

\author{
Bernhard Werner \\ Biologische Anstalt Helgoland (Zentrale); Notkestr. 31, D-2000 Hamburg 52, \\ Bundesrepublik Deutschland
}

\begin{abstract}
Further studies on the morphology, distribution and ecology of Stephanoscyphus planulophorus (Scyphozoa, Coronata). The solitary scyphopolyp Stephanoscyphus planulophorus Werner, 1971 was detected in the submarine caves of the French Mediterranean coast near Marseille, and on the Sorrent Peninsula as well as near the Isle of Ischia (Italy). It was rare at the French sites studied but in the Italian caves of the upper sublittoral it proved fairly abundant. Its life history is unique because (a) the medusa generation is reduced to a transitional embryonic stage, and (b) no germ cells could be traced in any phase. The polyps reared in the laboratory gave rise to several continuous generations. Thus, previous observations on their morphology, distribution and ecology could be completed. Growth and size (defined as length of the peridermal tube) are analyzed, and calculations of the form quotient $\mathrm{D} / \mathrm{L}(\mathrm{D}=$ upper diameter, $\mathrm{L}=$ length of tube) are given. Observations on the structure of peridermal teeth which mark the inner wall of the tube in its basal parts indicate that (a) shape is a characteristic of diagnostic value and (b) number and symmetrical arrangement can be described by the formula $4 \mathrm{P}+4 \mathrm{I}+8 \mathrm{~A}(\mathrm{~B} ; 5-7)$. A discontinuous circle of whitish pigment spots restricted to the endodermal cells of tentacle bases is another mark of diagnostic value confined to the soft body. S. planulophorus has a bicnidom of holotriches isorhizas and heterotrich microbasic euryteles. Size and distribution of nematocysts are listed for the life-cycle phases. The difference in distribution and population density between the sites near Marseille and Naples can be explained by local differences of water temperature and the thermal requirements of the polyp, the strobilation phase of which is temperature-sensitive. According to the present state of knowledge, S. planulophorus is one of the few true cave-living scyphopolyps. Considering the evolutionary consequences, the particulars of its life cycle can be interpreted as an adaptation to the special habitat conditions. The evolutionary progress of medusa reduction as well as the disadvantage of apogamous propagation are discussed briefly. A diagnosis is given based on the new morphological observations.
\end{abstract}

\section{EINLEITUNG}

Stephanoscyphus ist der Polyp der Coronata, der Basisordnung der Scyphozoa. Sein besonderes Kennzeichen, das ihn von den Polypen der Semaeostomea und Rhizostomea unterscheidet, ist der Besitz der vollständigen Peridermröhre.

Als Scyphopolyp erzeugt Stephanoscyphus die Medusen in der bekannten Weise durch Strobilation: Der obere Teil des Polypenkörpers wandelt sich durch Querteilung in Medusenanlagen um, die sich ablösen und als Jungmedusen (Ephyren) aus der Röhre ausschlüpfen und während der anschließenden planktischen Phase zur Geschlechtsreife 
heranwachsen. Die polydiske Form der Strobilation kann für Stephanoscyphus als typisch gelten (Werner 1966, 1967).

Die Strobilation galt bei den Scyphozoa lange Zeit als einzige Form der Medusenbildung. Als Ausnahme war lediglich die hypogenetische Entwicklung der Pelagiidae, Semaeostomea, bekannt, bei denen die Polypengeneration in Anpassung an die holopelagische. Lebensweise vollständig reduziert ist; hier folgt eine Medusengeneration direkt auf die andere. Später haben dann Komai \& Tokuoka (1939) und in neuerer Zeit hat Werner $(1971,1974)$ Stephanoscyphus-Arten beschrieben, deren Lebenszyklen ähnlich wie bei zahlreichen Hydroiden (Kühn, 1910, 1913) - durch eine Medusenreduk-

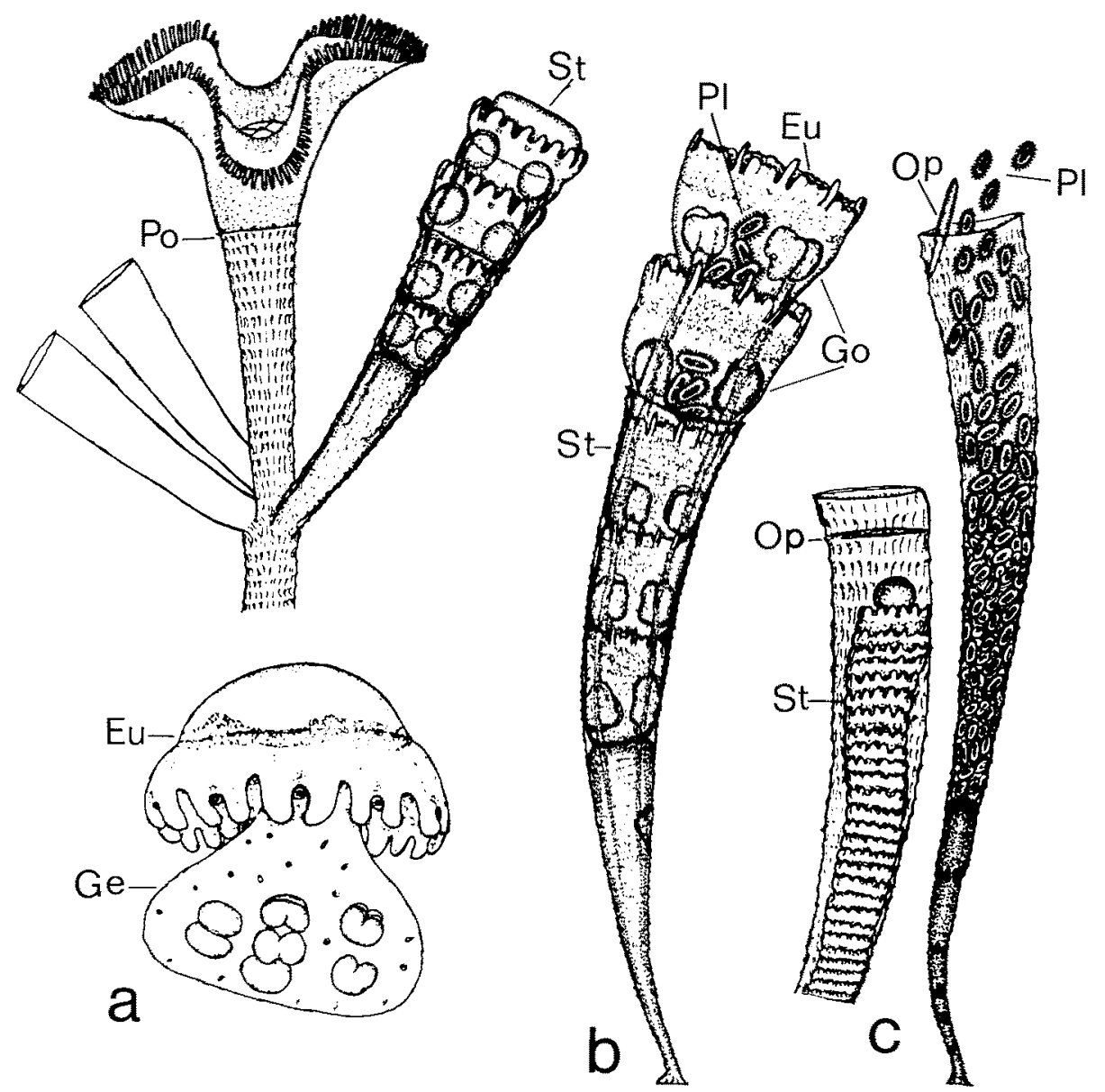

Abb. 1a-c. Stephanoscyphus. Evolutionsreihe der Medusenreduktion. (a) S. racemosus; auf der rechten Seite des Zweigstückes (oben) eine Strobila mit 4 Eumedusoiden mit reifen Eizellen. Unten freies Eumedusoid, das die in Gallerte eingebetteten Eizellen ausgestoßen hat; sie beginnen, sich zu furchen. (b) $S$. eumedusoides. Strobila mit 5 Eumedusoiden, die im Gastralraum zahlreiche Planulae enthalten. (c) S. planulophorus. Links Oberteil einer Strobila mit zahlreichen Anlagen von Ephyren. Rechts Polyp am Ende der Proliferationsphase mit zahlreichen ausschlüpfenden Planulae, die sich direkt aus den Ephyren entwickelt haben. Eu = Eumedusoid, Ge = Gallerte mit Eizellen, $\mathrm{Go}=$ Zwittergonade, $\mathrm{Op}=$ Operculum, $\mathrm{Pl}=$ Planulae, $\mathrm{Po}=$ Polyp, $\mathrm{St}=$ Strobila 
tion gekennzeichnet ist; sie unterscheiden sich dadurch von der Mehrheit der metagenetischen Scyphozoa. Wie bei den Hydroida lassen sich die Arten nach dem zunehmenden Grad der Reduktionserscheinungen zu einer evolutionistischen Reihe anordnen.

(a) Die stockbildende, reich verzweigte Species S. racemosus Komai, 1936 erzeugt durch oligodiske Strobilation getrenntgeschlechtige, kurzlebige Eumedusoide. Sie werden mit Keimzellen ausgestattet, die bereits in den Septen des Polypen heranreifen. Die männlichen Medusoide bleiben sessil und stoßen die Gameten ins freie Wasser aus. Die weiblichen Medusoide lösen sich noch ab, sind aber schwimmunfähig. Wie die Medusen der metagenetischen Arten sind sie ovipar. Die Eizellen werden in einer Gallertmasse ausgestoßen, in der sich die Entwicklung bis zur Planula vollzieht (Abb. 1a). S. racemosus ist an den Südostküsten von Japan stellenweise häufig und ist eine Form des obersten Sublitorals (5-10 m Wassertiefe).

(b) S. eumedusoides Werner, 1974 ist eine solitäre Form und erzeugt ebenfalls durch oligodiske Strobilation Eumedusoide, die aber sessil und mit dem basalen Restkörper des Polypen in ständiger Verbindung bleiben. Überdies sind sie zwittrig und larvipar: Die Eizellen entwickeln sich im Gastralraum zu Planulae, die beim anschließenden Zerfall der Strobilationskette frei werden (Abb. 1b). S. eumedusoides ist in den submarinen Felshöhlen der französischen Mittelmeerküste bei Marseille nicht selten. Wie ersichtlich, ist die Medusengeneration bei (a) und (b), wenn auch in reduzierter Form, erhalten und als Geschlechtsgeneration in voller Funktion.

(c) Zusammen mit $S$. eumedusoides wurde die gleichfalls solitäre Art S. planulophorus Werner, 1971 entdeckt, ist aber im gleichen Biotop sehr selten. Der Lebenszyklus dieser Art ist in mehrfacher Hinsicht ungewöhnlich (Abb. 1c). Die Medusenbildung durch polydiske Strobilation beginnt in der normalen, für die solitären Arten bekannten Weise (vgl. die Beschreibung der Einzelphasen bei Werner, 1966, 1967). So entsteht in der durch ein Peridermoperculum verschlossenen Röhre eine lange Kette von zahlreichen Medusenanlagen, die durch 8 Paare von Randlappen und durch 8 Statocysten eindeutig als Ephyren erkennbar sind. Sogar Muskelkontraktionen, wie sie auch bei den jungen Ephyren der metagenetischen Arten vor der Ablösung charakteristisch sind, lassen sich beobachten. Die Glieder der Strobilationskette beenden jedoch ihre Medusenentwicklung vorzeitig. Die Kette zerfällt in ihre Einzelanlagen, die sich kontinuierlich direkt in begeißelte Planulae umwandeln. Diese treten anschließend ins freie Wasser aus, heften sich nach einer kurzen planktischen Phase an einem geeigneten Substrat an und wandeln sich in der üblichen Weise in Primärpolypen um; damit nimmt eine neue Generation ihren Anfang.

S. planulophorus ist damit die erste Art, bei der die Medusengeneration im Endeffekt vollständig unterdrückt ist; sie erscheint in den Ephyrenanlagen nur noch als temporäre Ubergangsphase. Ihre Existenz läßt sich im Sinne der biogenetischen Grundregel Haeckels deuten. Was aber diesen Entwicklungsgang so besonders auffallend macht, ist das völlige Fehlen von Keimzellen, so daß von Anfang an mit der im gesamten Tierreich äußerst seltenen apogamen Entwicklung gerechnet werden mußte.

Die Erstbeschreibung basiert auf 4 Exemplaren, die 1969 aus einem Material von mehreren 100 Polypen der Art $S$. eumedusoides (s. oben) isoliert und in Laboratoriumsdauerkultur genommen waren. Bei der Suche nach Lebendexemplaren einer anderen neuen, Stephanoscyphus ähnlichen Art, die 1964 in untermeerischen Höhlen von Sorrent gefunden, aber erst später mit wenigen konservierten Tieren zur Kenntnis und 
Untersuchung gelangte, wurde 1975 in den gleichen Höhlen ein unerwartet reiches Vorkommen von $S$. planulophorus éntdeckt, so daß für die späteren Lebenduntersuchungen im Laboratorium ein reichhaltiges Material aller Alters- und Größenklassen zur Verfügung stand. Dabei wurden alle Eigenschaften des ungewöhnlichen Entwicklungsganges vollauf bestätigt, insbesondere das Fehlen einer Sexualphase mit der Bildung von Keimzellen. S. planulophorus ist daher eine Art mit apogamer Entwicklung.

Bei der Lebenduntersuchung über mehrere Jahre gelangten auch zahlreiche erwachsene Polypen zur Untersuchung. Sie ergab, daß die Erstbeschreibung, für die nur wenige kleine, daher nur junge Exemplare zur Verfügung standen, mehrere morphologische Eigenschaften unberücksichtigt gelassen hat, die auch von diagnostischem Wert sind. Der folgende Beitrag soll diese Lücken ausfüllen und überdies die Kenntnis dieser durch ihren ungewöhnlichen Entwicklungsgang interessanten Art auch hinsichtlich ihrer Verbreitung und Ökologie ergänzen.

Die genaue histologische und elektronenmikroskopische Untersuchung aller Phasen des Lebenszyklus, insbesondere der Bildung der Ephyren, ihrer direkten Umwandlung in Planulae, verbunden mit dem Fehlen von Keimzellen, wurde auf meinen Vorschlag von der Arbeitsgruppe Prof. M. Hündgen (Zoologisches Institut Bonn) und als Dissertationsthema von Herrn J. Hentschel übernommen. Ein gemeinsamer Bericht über die vorläufigen Ergebnisse ist in Vorbereitung (Werner \& Hentschel, 1983).

\section{MẢTERIAL UND METHODE}

Die im folgenden mitgeteilten Untersuchungen wurden an dem Freilandmaterial durchgeführt, das während eines Studienaufenthaltes an der Zoologischen Station Neapel im Mai 1975 in den untermeerischen Höhlen bei Sorrent während mehrerer Tauchgänge gesammelt war (vgl. Abb. 5); die Polypen fanden sich hauptsächlich an Stöckchen lebender und toter Korallen angeheftet und wurden im Laboratorium mit Hilfe eines Stereomikroskops abgesucht. Später standen dann auch die Kulturpolypen zur Verfügung, die von dem Marseiller und Sorrenter Ausgangsmaterial gezüchtet waren. Die Laboratoriumskultur von S. planulophorus über den gesamten Lebenszyklus bereitet keine Schwierigkeit, weil die langwierige Aufzucht der Medusengeneration wegfällt und als Anfangsglieder der Entwicklungsreihen sofort die Planulae zur Verfügung stehen. Sie heften sich an sauberen Stücken von Muschelschalen oder auch an Röhrenteilen von Polypen der gleichen Art oder anderer Arten an und lassen sich leicht aufziehen. Für die Kulturmethoden kann auf die kurze Darstellung bei Werner (1968) verwiesen werden, die nur wenig variiert wurden. Die Verwendung des Harpacticiden Tisbe holothuriae, der seit Jahren in Massenkulturen gezüchtet wird und sich als Futterorganismus bewährt hat, erleichtert die Dauerkultur erheblich. Vor allem erlaubt sie die leichte Aufzucht der aus den Planulae entstandenen winzigen Primärpolypen, denen die Nauplien des Harpacticiden wegen ihrer geringen Größe $(\sim 0,08 \mathrm{~mm})$ eine geeignete Nahrung sind.

Um die heranwachsenden Polypen zur regelmäßigen Strobilation zu bringen, empfiehlt sich die zusätzliche Einzelfütterung mit Stückchen von Mytilus-Mitteldarmdrüse in frischem Zustand, die ebenfalls seit Jahren als geeignete Nahrung für Cnidarier aller Klassen und Entwicklungsstadien erprobt ist. 


\section{ERGEBNISSE UND DISKUSSION}

\section{Ergänzende Beschreibung des Habitus und morphologischer Merkmale}

Bei der Aufklärung des vollständigen Lebenszyklus erwies sich Stephanoscyphus planulophorus als neue Art. Die Reduktion der Meduse schränkt die Darstellung der wesentlichen Artmerkmale auf die Polypengeneration ein. So ist S. planulophorus eine der vorerst noch wenigen Species, bei denen die lückenIose Artbeschreibung möglich ist und sich auf die Untersuchung der Merkmale des Habitus, der Peridermröhre und des Weichkörpers stützen kann.

\section{Habitus}

S. planulophorus gehört zu den obligatorisch solitären Formen. Indes fanden sich unter dem Sorrenter Material Aggregationen, in denen zahlreiche Tiere zu Pseudokolonien vereinigt waren. Auf den ersten Blick gleichen sie den echten Kolonien der Art Linuche unguiculata (Schwartz, 1788; syn. Stephanoscyphus komaii Leloup, 1937; vgl. Werner, 1979, p. 85), bei denen die unverzweigten Einzelpolypen von einer gemeinsamen Scyphorhiza ausgehen und erzeugt werden. Die Epithelien und Gastralräume aller Polypen stehen bei dieser Art durch die Basalplatte in ständiger Verbindung. Bei den Pseudokolonien von S. planulophorus aber handelt es sich lediglich um Aggregationen von Polypen, die sich am gleichen Substrat dicht nebeneinander oder auch an den Haftscheiben oder Basalteilen der Röhre ihrer Artgenossen angeheftet hatten. Die Weichkörper aller Einzeltiere bleiben daher ständig getrennt. Das läßt sich leicht nachweisen, wenn eine derartige Pseudokolonie in ihre Einzeltiere zerlegt wird. Außerdem ließ sich ihr Entstehen in den Kulturen mehrfach direkt beobachten. Wenn sich die Planulae in Aggregationen anhefteten, nahmen diese beim weiteren Wachstum der Polypen das gleiche doldenartige Aussehen an, wie es für die echten Kolonien von Linuche unguiculata charakteristisch ist. Es zeigte sich immer wieder, daß die Röhren älterer Polypen oder das Substrat in ihrer unmittelbaren Nachbarschaft von den Planulae bei der Anheftung deutlich bevorzugt wurden. Daraus ist zu schließen, daß die Planulae bei der Platzwahl auf artspezifische Substanzen reagieren, die von den Artgenossen ausgeschieden werden.

\section{Peridermröhre}

Wie erwähnt, ist Stephanoscyphus der einzige Scyphopolyp mit einer festen Peridermröhre aus Chitin, die den Weichkörper vollständig umgibt und aus der er nur mit dem Kopfteil und der Tentakelkrone herausschaut. Bei der Konservierung ohne vorherige sorgfältige Betäubung zieht sich der Körper so weit in die Röhre zurück und kontrahiert sich so stark, daß keinerlei Möglichkeit übrigbleibt, seine Organisation zu erkennen oder ihn auf Struktureigentümlichkeiten oder gar Artunterscheidungsmerkmale zu prüfen. In diesem Zustand befindet sich das meiste Material, das in den Museen angesammelt ist. So könnten auch Schnittuntersuchungen kaum Klarheit über Körperbau oder Entwicklungsgänge bringen. Daher kommt der Untersuchung der Röhrenmerkmale besondere Bedeutung zu, auch wenn die Ermittlung der Artzugehörigkeit ohne Kenntnis der Weichkörperorganisation und des gesamten Lebenszyklus einschließlich der Medusengeneration nach den vorliegenden Erfahrungen in den meisten Fällen schwierig oder unmöglich ist. 
Die Zahl der Arbeiten, in denen versucht wird, Stephanoscyphus-Arten auf Grund der Röhrenstrukturen gegeneinander abzugrenzen, ist gering. Bei der Bearbeitung des während der dänischen "Galathea"-Expedition 1950-1952 gesammelten Materials, das aus ganz verschiedenen Zonen und Wassertiefen stammte, gelangte Kramp $(1959,1962)$ zur angenäherten Kennzeichnung lediglich zweier solitärer Arten, nämlich von $S$. simplex Kirkpatrick, 1890 und $S$. corniformis Komai, 1936. Die seitdem durch die eigenen Untersuchungen gewonnene Erfahrung, daß sich hinter diesen Namen zahlreiche verschiedene Arten, mehrere Gattungen und sogar Familien verbergen, dürfte auch für das "Galathea"-Material zutreffen. Daher kann man bisher bei der Identifizierung konservierten Materials höchstens zu einer Zuordnung zur S. simplex-Gruppe oder $S$. corniformis-Gruppe gelangen. Dementsprechend hat Naumov (1959), dem konserviertes Material aus dem sibirischen Eismeer vorlag, die von ihm unterschiedenen Arten konsequenterweise als $S$, spec, $1,2,3$ vorgestellt.

Für die allgemeinen Merkmale der Peridermröhre (Form, Größe, Farbe, Oberflächenskulptur, Vorhandensein und Beschaffenheit der Zahnbildungen: vgl. Komai, 1936; Leloup, 1937; Kramp, 1959; Naumov, 1959; Werner, 1966, 1967) kann auf die Erstbeschreibung verwiesen werden (Werner, 1971, p. 125 f.). Die vergleichende Prüfung an dem reichhaltigen Material aus den Sorrenter Höhlen erlaubt es, die Angaben zur Form und Größe sowie der Art der Zahnbildungen durch quantitative Daten und genauere Beobachtung zu präzisieren.

\section{Form und Größe}

Die Röhre hat eine schlanke, leicht gebogene Form, die sich von der Basis bis zur oberen Mündung schwach verbreitert. Die Farbe des Chitinmaterials ändert sich von einem kräftigen Braun der unteren Teile mit verdickter Röhrenwandung zu einem hellen Weißgelb der dünnwandigen Röhrenmündung. Die fast gerade Idealform läßt sich in Einzelkulturen beobachten, wenn sich eine Planula am Glasboden oder an einem ebenen Substrat anheftet, so daß der Polyp nach der Umwandlung frei nach oben wachsen kann. In der natürlichen Umgebung sind die Polypen vielfach zu mehr oder weniger dichten Aggregationen vereinigt. Sie sind dann gezwungen, der Form, den Unebenheiten und Unregelmäßigkeiten des Substrats zu folgen oder solchen auszuweichen sowie sich in die oft bündel- oder doldenförmige Anordnung der Artgenossen einzupassen. Die Röhre nimmt daher bei den meisten Exemplaren mit zunehmendem Alter eine stärker gebogene, einfach, mehrfach oder unregelmäßig gekrümmte Form an.

Gegenüber dem Scyphistoma der Semaeostomea und Rhizostomea, bei dem die Größe wegen der Reduktion der Röhre vom Weichkörper bestimmt wird und mit dessen Kontraktionszustand wechselt, bietet Stephanoscyphus den Vorteil, daß die Länge der stabilen Röhre ein zuverlässiges Maß für die Größe und die Wachstumsgeschwindigkeit liefert. Wie früher beschrieben (Werner, 1970, 1979), ist die Größe der Einzelpolypen bei den verzweigten, stockbildenden Arten begrenzt und überschreitet eine für die betreffende Art genetisch fixierte Größe nicht. Durch den solitären Habitus gehört S. planulophorus zu den Arten, die ein ständiges Längenwachstum aufweisen. Die in der natürlichen Umgebung gefundenen Polypen haben meist nur eine geringe Größe und überschreiten selten eine Länge von $1 \mathrm{~cm}$. Uber ihr Alter kann nichts ausgesagt werden. Das in den Höhlen vermutlich geringe Nahrungsangebot und die Nahrungskonkurrenz der Artgenossen oder der anderen Epizoen verhindern offenbar die volle Entfaltung der $S$. 
planulophorus innewohnenden Wachstumspotenzen. Denn die Kulturpolypen, die über Jahre hinweg unter guten Nahrungsbedingungen leben, erreichen ein sehr viel größere Länge (Tab. 1). Das Wachstum wird zwar im Alter verlangsamt, wird aber nicht völlig beendet, wie die unmittelbare Beobachtung erkennen ließ.

Tab. 1. Stephanoscyphus planulophorus, quantitative Daten der Peridermröhre. $\mathbf{n}=$ Anzahl untersuchter Röhren, $L=$ Länge, $D=$ oberer Durchmesser, $Q=$ Formquotient $D / L, u D=$ unterer Durchmesser, Hs $=$ Durchmesser der Haftscheibe, $\mathrm{Z}=$ Zahl der Zahnkränze. Mittelwerte \pm Standardabweichungen

\begin{tabular}{|cccccccc|}
\hline $\begin{array}{c}\text { Größen- } \\
\text { klassen } \\
(\mathrm{mm})\end{array}$ & $\mathrm{n}$ & $\mathrm{L}$ & $\mathrm{D}$ & $\mathrm{Q}$ & $\mathrm{uD}$ & Hs & $\mathrm{Z}$ \\
\hline & & $(\mathrm{mm})$ & $(\mathrm{mm})$ & & $(\mathrm{mm})$ & $(\mathrm{mm})$ & \\
\hline 20,0 & 17 & $24,91 \pm 4,03$ & $1,16 \pm 0,13$ & 0,05 & $0,19 \pm 0,03$ & $0,36 \pm 0,09$ & $6,19 \pm 0,66$ \\
$15,0-19,9$ & 28 & $16,56 \pm 1,11$ & $1,14 \pm 0,12$ & 0,07 & $0,21 \pm 0,04$ & $0,48 \pm 0,13$ & $5,89 \pm 1,01$ \\
$10,0-14,9$ & 52 & $12,20 \pm 1,70$ & $1,02 \pm 0,12$ & 0,08 & $0,20 \pm 0,04$ & $0,46 \pm 0,14$ & $5,62 \pm 0,97$ \\
$5,0-9,9$ & 36 & $8,12 \pm 1,36$ & $0,85 \pm 0,15$ & 0,10 & $0,21 \pm 0,06$ & $0,44 \pm 0,11$ & $5,14 \pm 0,93$ \\
$\leqslant 4,9$ & 2 & $3,25 \pm 0,49$ & $0,40 \pm 0,0$ & 0,12 & $0,23 \pm 0,04$ & $0,45 \pm 0,21$ & $2,00 \pm 0,0$ \\
\hline
\end{tabular}

Nach der schlanken Grundform der Röhre gehört S. planulophorus der S. corniformis-Gruppe an. Als quantitatives Maß hat Kramp (1959) den Quotienten $Q=D / L$ aus oberem (= größtem) Durchmesser (D) und Länge der Röhre (L) eingeführt. Werden außerdem als weitere Maße der untere (kleinste) Durchmesser der Röhre dicht oberhalb der Haftscheibe und der Durchmesser dieser selbst bestimmt, so läßt sich die Form der Röhre einigermaßen quantitativ beschreiben. Die Auswertung der Meßdaten (Tab. 1) führt zu folgenden Resultaten:

(a) Der Durchschnittswert des Formquotienten für alle Größen (135 Exemplare) beträgt 0,07 .

(b) Das relative Wachstum der Röhre ist dadurch gekennzeichnet, daß das Längenwachstum positiv allometrisch zum Dickenwachstum ist. Letzteres verlangsamt sich mit zunehmender Länge und zunehmendem Alter. Daher ist der Formquotient bei den kleineren und größeren Tieren verschieden; er verringert sich deutlich mit zunehmender Länge und nimmt bei den angegebenen Größenklassen von 0,12 bis 0,05 ab.

(c) Dabei ist zu berücksichtigen, daß der Formquotient ein empfindlicher Indikator für die Abhängigkeit des Wachstums von äußeren Einflüssen, speziell von der Ernährung, ist. Bei mangelhafter Ernährung wird das Dickenwachstum stärker beeinflußt als das Längenwachstum, so daß die Röhren mit zunehmender Länge wieder dünner werden können.

(d) Der untere Durchmesser der Röhre dicht über der Haftscheibe ist eine annähernd konstante Größe. Das läßt sich damit erklären, daß dieser älteste Teil der Röhre von dem Primärpolypen unmittelbar nach seiner Metamorphose aus der angehefteten Planula noch vor der Tentakelbildung und ersten Nahrungsaufnahme angelegt wird. Die Dimension des ältesten Teiles der Röhre hängt damit allein von der Menge der der Planula mitgegebenen Reservesubstanzen ab. Aus den geringen Größenunterschieden der Planulae läßt sich daher die Größenkonstanz des basalen Primärteiles der Röhre hinreichend erklären. 
(e) Das gleiche gilt für die Haftscheibe. Ihre Größe ist gering und verändert sich auch beim weiteren Wachstum des Polypen kaum noch. Der Durchmesser ist annähernd doppelt so groß wie der des Basalteiles der Röhre.

Das allometrische Wachstum der Röhre läßt sich auch in der Weise verifizieren, daß die Durchmesser ein und derselben Röhre in verschiedenem Niveaus von unten nach oben gemessen und in Beziehung auf die Höhe der Niveaueinteilung gesetzt werden. Dann zeigt sich wie bei der Einteilung in Größenklassen verschiedener Röhren, daß der Formquotient an der Röhrenbasis den größten Wert hat und kontinuierlich nach oben abnimmt.

\section{Zahnbildungen}

Bei den meisten Stephanoscyphus-Arten trägt die Innenwand der Röhre als Zähne oder Dornen bezeichnete hohle Vorsprünge, die beim Wachstum der Röhre nacheinander erzeugt werden, normalerweise in horizontalen Kränzen stehen und von unten nach oben mit dem Durchmesser der Röhre an Größe zunehmen. Die Entstehung der Zähne wurde früher beschrieben (Chapman \& Werner, 1972, p. 414). Bei vielen Arten besteht ein Zahnkranz aus 4 perradialen größeren und 4 interradialen kleineren Zähnen. Ihre Anordnung ist derart, daß die Zähne der gleichen Symmetrie bei den Kränzen verschiedener Röhrenniveaus in vertikalen Reihen übereinander liegen. Da die Zahnbildungen nicht bei allen Arten gleichförmig sind, da vielmehr auch ihre Zahl vermehrt oder reduziert sein kann, da sie überdies auch völlig fehlen können, sind sie von relativer systematischer Bedeutung, so daß sie auch in der Diagnose Berücksichtigung finden müssen.

Wie früher beschrieben, besitzt $S$. planulophorus in jedem Zahnkranz 16 Zähne: 4 perradiale (P), 4 interradiale (I) und 8 adradiale Zähne (A). Daher läßt sich die Beschreibung durch eine Zahnformel vereinfachen; sie lautet für unsere Art: $4 \mathrm{P}+4 \mathrm{I}+8 \mathrm{~A}_{\mathrm{i}}$ sie würde bei den anderen Arten mit 8 Zähnen $4 \mathrm{P}+4 \mathrm{I}$ lauten. Bei den Arten mit vollständigen Zahnkränzen werden meist mehrere Zahnkränze angetroffen,
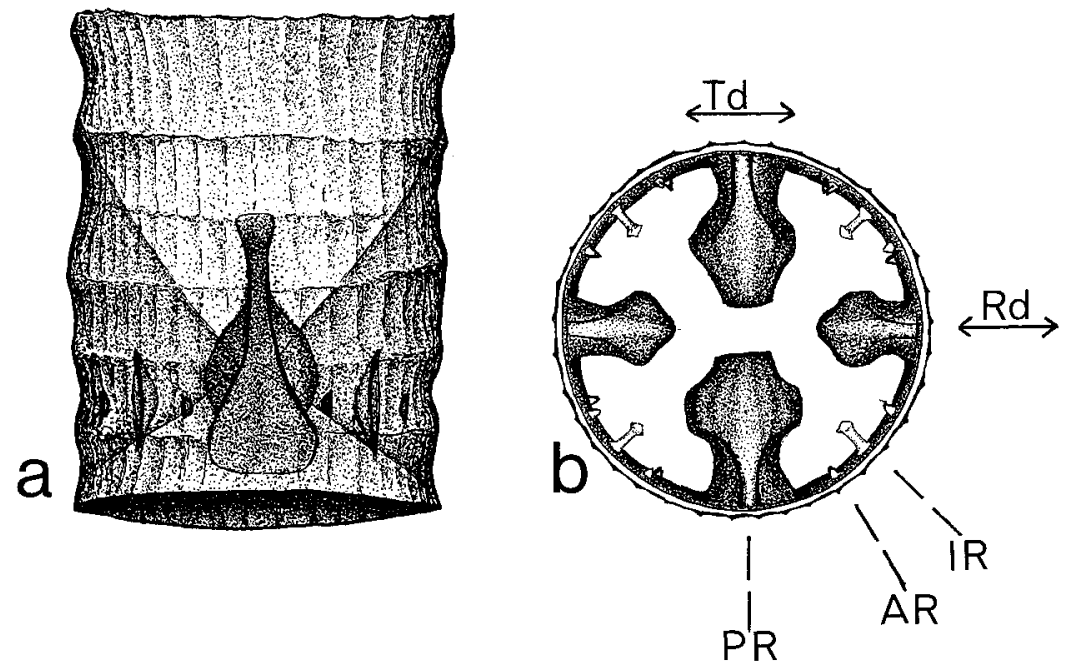

Abb. 2a, b. S. planulophorus. Röhrenstück mit Zahnkranz, (a) Seitenansicht, (b) Aufsicht von oben. $\mathrm{Rd}=$ radialer, $\mathrm{Td}=$ tangentialer Durchmesser eines Zahnes. $\mathrm{AR}=$ Adradius, IR $=$ Interradius, $\mathrm{PR}=$ Perradius. (Nach Werner, 1971, verändert) 
die entweder auf den basalen Teil (B) oder auf die Gesamtlänge der Röhre (R) verteilt sind. Bei den Röhren größerer Polypen ( $\geqq 10 \mathrm{~mm}$ ) von $S$. planulophorus (Tab. 1) sind meist 5-7 Zahnkränze vorhanden, die auf etwa die unteren $2 / 5$ oder $1 / 2$ der Röhre beschränkt sind. Die vollständige Zahnformel lautet daher $4 \mathrm{P}+4 \mathrm{I}+8 \mathrm{~A}(\mathrm{~B} ; 5-7)$.

Weiterhin bestehen bei den Arten von Stephanoscyphus Unterschiede in der Form der Zähne. Ihr Umriß kann in der Aufsicht von oben oder unten glatt oder gezackt und mit Spitzen versehen sein. Daß derartige Formunterschiede nur relativ brauchbar sind, ergibt sich aus der Beobachtung, daß bei der gleichen Röhre die älteren Zähne eines in einem tieferen Niveau gelegenen Zahnkranzes einen unregelmäßig gezackten, die jüngeren Zähne eines höheren Niveaus aber einen glatten Umriß aufweisen können. Außerdem können ihr tangentialer und radialer Durchmesser sowie die Form des Ansatzes an der Röhrenwandung unterschiedlich sein.

Bei der Uberprüfung der früheren Angaben über Form und Größenunterschiede der

Abb. 3a-d. S. planulophorus. Variation der Form eines großen perradialen Zahnes der Zahnkränze der verschiedenen Niveaus bei der gleichen Röhre (vgl. Abb. 2a). Das Alter nimmt von unten nach oben ab. (a) aus dem Zahnkranz 2 nahe der Röhrenbasis, (b) aus dem Zahnkranz 5, (c) aus dem Zahnkranz 6, (d) aus dem jüngsten, obersten Zahnkranz 8. Die vertikalen Linien des Zahnansatzes an der Röhrenwand dick gezeichnet

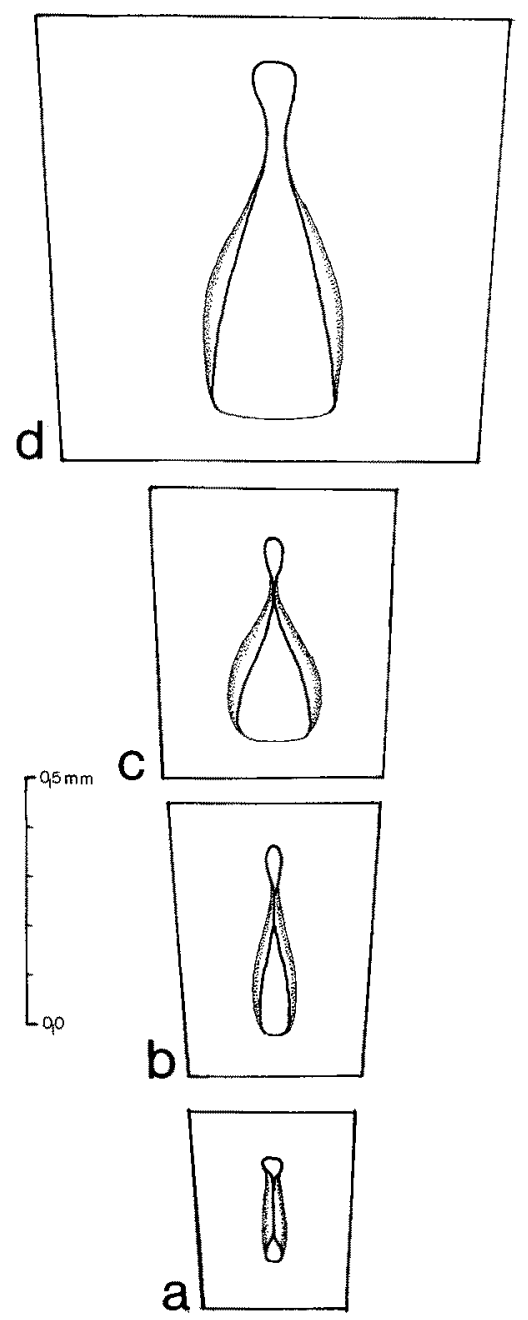


Zähne eines Zahnkranzes von S. planulophorus (Werner, 1971, p. 125, Abb. 2a, b) zeigte sich, daß sie in einem Punkt der Ergänzung bedürfen. Es betrifft das im vorhergehenden zuletzt erwähnte Merkmal der Form des Ansatzes der Zahnbasis an der Röhrenwandung. Die besondere Form des Zahnansatzes ist vor allem bei den großen perradialen Zähnen deutlich, weniger bei den kleinen interradialen; bei den winzigen adradialen Zähnen ist dieses Merkmal bedeutungslos.

Die Darstellung eines Kranzes in der Seitenansicht und im Querschnitt (Abb. 2) läßt die charakteristisch schmale Form des Ansatzes der Zahnbasis deutlich erkennen; er ist basal etwas erweitert und verschmälert sich nach oben, das heißt in Richtung auf die Röhrenmündung. Die Ansatzlinien laufen hier fast zusammen. Die Dicke (= der tangentiale Durchmesser) eines solchen Zahnes ist daher an seinem Ansatz geringer als in seiner Mitte, wo er blasig erweitert ist. Das kommt in der Aufsicht auf den Zahnkranz von oben deutlich heraus (Abb. 2b).

Indes zeigt der Vergleich der unteren und oberen Zahnkränze bei der gleichen Röhre, daß die Art des Zahnansatzes nicht konstant ist, daß er sich vielmehr mit der Größe der Zähne ändert. Wie die Abb. 3a-d zeigt, liegen die vertikalen Linien des Zahnansatzes bei den unteren, kleineren und älteren Zähnen dicht zusammen und weichen bei den oberen, größeren und jüngeren in zunehmendem Maße auseinander, so daß der in Abb. 2a dargestellte Zustand der oberen Zahnkränze erreicht wird. Das beschriebene Merkmal der Art des Zahnansatzes in der geschilderten, alters- und größenbedingten Variationsbreite ist für S. planulophorus charakteristisch und ermöglicht die Identifizierung gegenüber den anderen bisher aus dem Mittelmeer bekannten Stephanoscyphus-Arten.

\section{Weichkörper}

Auch die frühere Beschreibung der Weichkörperstrukturen kann jetzt durch ein qualitatives Merkmal ergänzt werden. Es ist so charakteristisch, daß es bei lebenden Polypen die sofortige Identifizierung erlaubt, weil es von keiner anderen Mittelmeerart

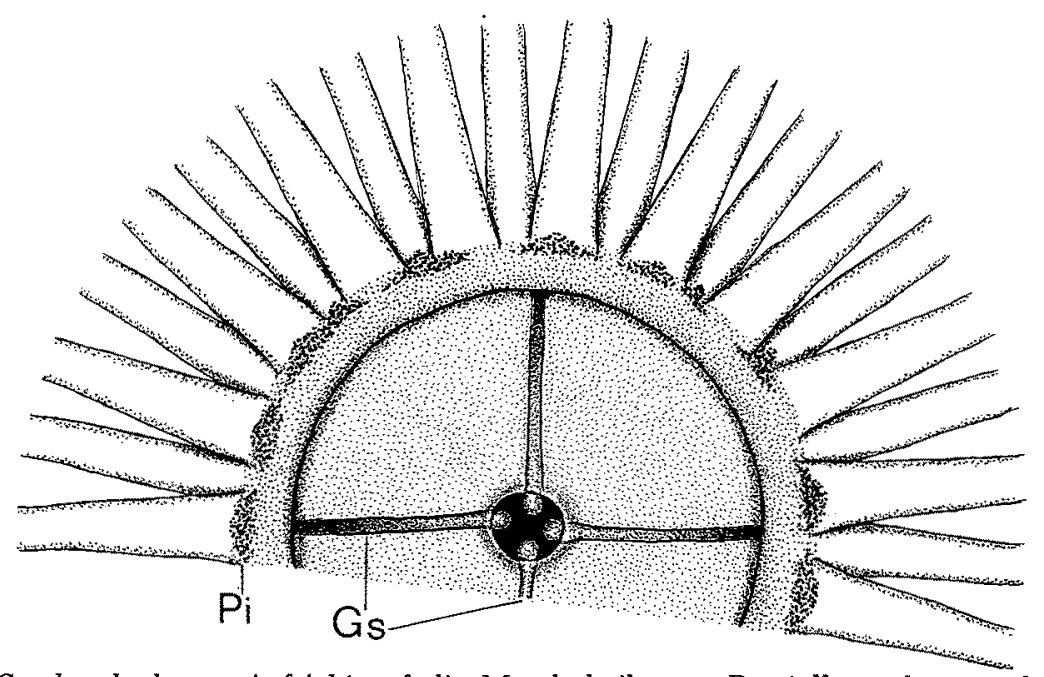

Abb. 4. S. planulophorus, Aufsicht auf die Mundscheibe zur Darstellung des unterbrochenen Pigmentringes an der Basis der Tentakel. Beachte den radial versetzten Ansatz der Tentakel. Gs $=$ Gastralsepten, die an der Mundöffnung zusammenlaufen, $\mathrm{Pi}=$ Pigmentfleck 
bekannt ist. Bei $S$. planulophorus läßt sich nämlich an der Basis der Tentakel ein nicht sehr auffallendes, aber doch deutliches Farbmuster erkennen (Abb. 4, Aufsicht). Es handelt sich um schmale horizontale Flecken von weißgelblicher, im Auflicht schwach grünlicher Färbung. Wie die nähere Beobachtung zeigt, tragen nicht alle Tentakel diesen schmalen Pigmentstreifen. Vielmehr wechselt ein Tentakel mit einem Farbstreifen mit einem benachbarten ohne Pigmentierung ab, wobei allerdings Unregelmäßigkeiten auftreten können, so daß von benachbarten Tentakeln beide oder keiner die Farbflecken trägt. Meist aber ist die Zahl der Einzelflecken des unterbrochenen Pigmentringes am Ansatz der Tentakel etwa gleich der Hälfte der Tentakelzahl (Tab. 2). Der Grund dafür, daß dieses charakteristische Merkmal bei den 4 Polypen der Erstbeschreibung nicht bemerkt wurde, ist darin zu suchen, daß es bei jungen Polypen noch fehlt oder so schwach ausgeprägt ist, daß es leicht übersehen werden kann. Da die histologische Untersuchung noch aussteht, kann über die cytologische Grundlage lediglich gesagt werden, daß die Farbpigmente in der Form von kurzen Nadeln vorliegen und im Entoderm der Tentakelbasis lokalisiert sind.

Quantitative Merkmale des Weichkörpers, die zwar nur einen begrenzten diagnostischen Wert haben, aber ohne die eine einwandfreie morphologische Darstellung unvollkommen wäre, sind Zahl und Länge der Tentakel sowie Breite des Kragens. Diese Merkmale können auch deswegen nur relativ sein, weil sie von der Größe der Tiere und ihrem jeweiligen Kontraktionszustand abhängen. Läßt man aber die Tiere vor der Untersuchung etwa eine Woche lang hungern, so kann man sicher sein, daß Kopfteil und Tentakel voll ausgestreckt sind (Tab. 2). So bestätigt sich für S. planulophorus, daß die Zahl der Tentakel mit der Größe variiert. Die Durchschnittszahl beträgt bei den größeren Tieren ( $\geqq 15 \mathrm{~mm}$ ) 30 bis 40 ; die bislang angetroffene Höchstzahl war 47 . Ferner geht aus den Messungen hervor, daß die Breite des Kragens bei den Tieren gleicher Größengruppe eine annähernd konstante Größe ist; sie beträgt 0,5 mm.

Tab. 2. Stephanoscyphus planulophorus, quantitative Daten des Weichkörpers lebender, vollausgestreckter Polypen, $\mathbf{n}=$ Anzahl der untersuchten Tiere. Mittelwerte \pm Standardabweichungen

\begin{tabular}{|c|c|c|c|c|c|c|}
\hline \multirow{2}{*}{$\begin{array}{c}\text { Größen- } \\
\text { klassen } \\
(\mathrm{mm})\end{array}$} & \multirow[t]{2}{*}{$\mathbf{n}$} & \multirow{2}{*}{$\begin{array}{l}\text { Länge* } \\
\text { (mm) }\end{array}$} & \multirow{2}{*}{$\begin{array}{c}\text { Kragenbreite } \\
\text { (mm) }\end{array}$} & \multicolumn{2}{|c|}{ Tentakel } & \multirow[b]{2}{*}{$\begin{array}{l}\text { Pigment- } \\
\text { flecken-- } \\
\text { zahl }\end{array}$} \\
\hline & & & & Zahl & $\begin{array}{l}\text { Länge ** }^{*} \\
\text { (mm) }\end{array}$ & \\
\hline$\geq 20,0$ & 10 & $25,00 \pm 3,54$ & $0,49 \pm 0,02$ & $36,10 \pm 4,36$ & $\begin{array}{l}6,3 \\
1,5\end{array}$ & $17,40 \pm 2,17$ \\
\hline $15,0-19,9$ & 2 & $15,90 \pm 0,57$ & $0,50 \pm 0,00$ & $34,50 \pm 3,54$ & $\begin{array}{l}4,8 \\
1,5\end{array}$ & $15,50 \pm 0,71$ \\
\hline $10,0-14,9$ & 5 & $12,56 \pm 1,77$ & $0,33 \pm 0,04$ & $35,75 \pm 4,50$ & $\begin{array}{l}1,5 \\
4,5 \\
1,2\end{array}$ & $18,50 \pm 0,71$ \\
\hline $5,0-9,9$ & 6 & $6,35 \pm 1,21$ & $0,27 \pm 0,08$ & $27,33 \pm 3,61$ & $\begin{array}{l}4,8 \\
1,2\end{array}$ & $13,20 \pm 3,11$ \\
\hline \multicolumn{7}{|c|}{$\begin{array}{l}\text { * Wie in Tab. } 1 \text { wird als definierte Länge die der Röhre angegeben. Die genaue Länge des } \\
\text { Weichkörpers ist um die Breite des Kragens zu vergrößerm. } \\
\text { * Wegen der unterschiedlichen Lage der Tentakel im Raum werden von den horizontal } \\
\text { ausgestreckten Tentakeln jeweils nur der kïrzeste und längste Tentakel angegeben. }\end{array}$} \\
\hline
\end{tabular}




\section{Cnidom}

Wie bereits früher mitgeteilt, besitzt $S$. planulophorus wie alle anderen Coronata (soweit bekannt) ein Bicnidom aus holotrichen Isorhizen (Haplonemen) und mikrobasischen heterotrichen Eurytelen (Heteronemen). Beim Polypen sind die Nesselzellen an den Tentakeln zu Batterien in der Form schmaler Flecken sowie unvollständiger Ringe oder Spiralen konzentriert, während die ganze Oberfläche des Kragens gleichmäßig von Nesselzellen besetzt ist. Die Größenverhältnisse und Verteilung sind in der Tabelle 3

Tab. 3. Stephanoscyphus planulophorus, Cnidom*. Größe (Länge $\times$ Breite) der unentladenen Nesselkapseln in $\mu \mathrm{m}$; häufigste Werte fettgedruckt. Zahl der Messungen in Klammern

\begin{tabular}{|c|c|c|}
\hline $\begin{array}{l}\text { Stadien und } \\
\text { Verteilung }\end{array}$ & $\begin{array}{c}\text { Haplonemen } \\
\text { Holotriche Isorhizen }\end{array}$ & $\begin{array}{c}\text { Heteronemen } \\
\text { Heterotriche mikrobasische } \\
\text { Eurytelen }\end{array}$ \\
\hline \multicolumn{3}{|l|}{ Polyp } \\
\hline \multirow[t]{2}{*}{ Kragen } & wenige, meist Bildungsstadien & sehr zahlreich \\
\hline & $5,6-7,5-8,8 \times 3,7-5,6-6,2(10)$ & $11,9-12,5-13,8 \times 10-11,2(10)$ \\
\hline \multirow[t]{2}{*}{ Tentakel } & sehr zahlreich & wenige, meist an der Basis \\
\hline & $6,2-8,1-8,7 \times 5,0-5,6-6,2(10)$ & $12,5-13,8 \times 10,0-10,6-11,2(10)$ \\
\hline \multicolumn{3}{|l|}{ Ephyra } \\
\hline Scheibe & wenige & $\begin{array}{l}\text { in peripherem Ring } \\
10,0-12,5 \times 7,5-9,4-11,2(10)\end{array}$ \\
\hline Randlappen & $\begin{array}{l}\text { zahlreich } \\
6,2-8,7 \times 3,8-5,0-5,6(10)\end{array}$ & wenige \\
\hline \multicolumn{3}{|l|}{ Planula } \\
\hline
\end{tabular}

zusammengestellt. Die Meßwerte lassen erkennen, daß die verschiedenen Stadien auch größenmäßig die annähernd gleiche Nesselzellausstattung haben. Das ist bei einem Vergleich des Polypen und der Ephyren nicht auffallend, da letztere ja durch einen Strobilationsprozeß aus ersterem hervorgehen. Ein geringer Größenunterschied besteht lediglich zwischen den Eurytelen der Ephyren und der aus ihnen metamorphosierten Planulae, bei denen sie deutlich kleiner sind. Daraus läßt sich schließen, daß sie bei der Planula neu gebildet werden. Beide Kapseltypen sind mit wohlentwickelten Cnidocils versehen, die aber eine signifikant verschiedene Länge haben: Beim Polypen haben die Cnidocils der Holotrichen eine Länge von $15 \mu \mathrm{m}$ bis $17,5 \mu \mathrm{m}$, die der Eurytelen aber nur von 3,75 bis $5,0 \mu \mathrm{m}$.

\section{Verbreitung und Ökologie}

Die Lage der untersuchten Höhlen der Felsküste bei Sorrent (Kalkstein) ist in Abb. 5 dargestellt, und ihre Eigenschaften sind durch die Angaben der Tabelle 4 kurz skizziert. 
Tabl. 4. Submarine Höhlen bei Sorrent und Ischia, die vom 14. bis 27. 5.1975 auf das Vorkommen von Stephanoscyphus-Arten untersucht worden sind (vgl. Abb. 5). L = Länge, $\mathrm{B}=\mathrm{Breite}, \mathrm{H}=$ Höhe $(\mathrm{m})$

\begin{tabular}{|c|c|c|c|}
\hline Name und Typ & $\begin{array}{l}\text { Tiefenlage } \\
(\mathrm{m})\end{array}$ & $\begin{array}{l}\text { Größe } \\
(\mathrm{m})\end{array}$ & $\begin{array}{l}\text { S. planulophorus } \\
\text { Fundzahlen }\end{array}$ \\
\hline \multicolumn{4}{|l|}{ Sorrent } \\
\hline Punta di San Antonio, & (a) 8 & L 40 & 180 \\
\hline 2 Sackhöhlen & (b) $10-15$ & L 80 & 673 \\
\hline Zaffiro, Sackhöhle & $5-10$ & L 80 & 117 \\
\hline Isola d'Isca, & $5-15$ & L 100 & \\
\hline Sackhöhle mit & & В $30-50$ & \\
\hline Luftkuppel & & $\mathrm{H} 30$ & 13 \\
\hline $\begin{array}{l}\text { Punta di San Elia, } \\
\text { Sackhöhle }\end{array}$ & $6-7$ & L 30 & 16 \\
\hline $\begin{array}{l}\text { Montalto, } \\
\text { Sackhöhle }\end{array}$ & 45 & $?$ & $\begin{array}{l}\text { wenige, } \\
\text { sehr klein }\end{array}$ \\
\hline \multicolumn{4}{|l|}{ Ischia } \\
\hline $\begin{array}{l}\text { Formiche di Vivara, Kuppe } \\
\text { (a) mit } 3 \text { Öffnungen }\end{array}$ & 15 & $\begin{array}{lr}\text { L } & 100 \\
\text { B } & 50\end{array}$ & 2 \\
\hline (b) Sackhöhle & 15 & $?$ & 19 \\
\hline
\end{tabular}

Die Höhlen liegen mit einer Ausnahme im oberen Sublitoral in Tiefen von 5 bis $15 \mathrm{~m}$. Sie gehören überwiegend dem Typ der geschlossenen Sackhöhle an, liegen also vollständig unter Wasser, haben nur einen Eingang und sind damit als Stillwasserzonen zu charakterisieren. Die Besiedlung der Wände und Decken durch Invertebraten ist in der Nähe des Höhleneinganges sehr dicht, und hier liegt auch die Zone mit der größten Besiedelungsdichte von $S$, planulophorus, denen die zahlreichen Korallenstöckchen das notwendige Substrat bieten. Die Tierbesiedelung nimmt im vorderen Höhlendrittel bis zur Mitte schnell ab und ist in den hinteren Teilen sehr spärlich. Eine Höhle (an der Isola d'Isca) hat eine größere Höhe und eine Luftkuppel über dem Meeresspiegel. Das bedingt eine stärkere Durchströmung, weil das Luftpolster je nach Wetterlage und äußerem Wasserstand als Pumpmechanismus wirkt. Eine stärkere Durchströmung erfolgt auch in den Höhlen mit mehreren Eingängen. Zu diesem Typ gehört eine der Höhlen von Formiche di Vivara bei Ischia.

Hinsichtlich der hydrographischen Verhältnisse ist wichtig, daß die Höhlen wegen der geringen Wassertiefe im Sommer oberhalb der thermischen Sprungschicht liegen, so daß die Temperaturen in den Höhlen $20^{\circ}$ überschreiten. Nur die Höhle von Montalto (45 m) liegt unterhalb der Sprungschicht und hat auch im Sommer niedrige Temperaturen. Eine Ausnahme machen auch die beiden Höhlen von Formiche di Vivara vor Ischia. Das Höhlensystem liegt nicht in der Felsküste, sondern in einiger Entfernung in einer flachen untermeerischen Kuppe, die bis auf 6-10 m unter der Wasseroberfläche ansteigt. 
Das in dieser Kuppe befindliche Höhlensystem gehört daher einem Horizont geringer Tiefe an. In der Nähe befindet sich jedoch eine tiefere Senke, aus der im Sommer durch die vorherrschende Nord-Süd-Strömung kälteres Wasser an die Oberfläche gebracht wird, so daß hier die Sprungschicht höher liegt.

Wenn auch Ermittlungen über die Bestandsdichte von $S$. planulophorus in den untersuchten Höhlen wegen der Kürze der verfügbaren Zeit nicht angestrebt waren, so zeigen die Fundzahlen der Tabelle 5 doch, daß die Besiedelung unterschiedlich und daß diese Art in einigen Höhlen sehr häufig war. Auffällig war besonders das sehr spärliche Vorkommen in der Höhle Montalto der größten Wassertiefe $(45 \mathrm{~m})$, wobei es sich um wenige, sehr kleine Exemplare handelte. Im ganzen belegen die Fundbeobachtungen das reiche Vorkommen in den Höhlen von Sorrent, was in eindrucksvollem Gegensatz zu dem sehr seltenen Auftreten in den Höhlen bei Marseille steht. Dort waren, wie erwähnt, unter mehreren hundert Polypen der verwandten Art $S$, eumedusoides (vgl. Werner, 1974) bei mehrjährigen Untersuchungen nur 4 Exemplare gefunden worden.

Wie erklärt sich der signifikante Unterschied der Bestandsdichte zwischen der nördlichen Mittelmeerküste bei Marseille und dem Golf von Neapel? Alle Beobachtungen sprechen dafür, daß die Ursache in den Temperaturansprüchen des Polypen und in den lokal verschiedenen Temperaturbedingungen zu suchen ist. Nach den langjährigen Kulturbeobachtungen ist $S$. planulophorus eine Wärmeform, und zwar ist die Strobilation die kritische, temperaturempfindliche Phase im Lebenszyklus. Bei Temperaturen von 20 bis $22^{\circ} \mathrm{C}$ ist ein regelmäßiger Wechsel zwischen Strobilations- und Regenerations- + Wachstumsphase zu beobachten ${ }_{r}$ und in diesem Temperaturbereich strobiliert der Polyp deutlich häufiger als bei niedrigeren Temperaturen. Die untere Grenze der Strobilationstemperatur liegt bei etwa $16^{\circ} \mathrm{C}$.

Der Vergleich mit den lokalen Temperaturbedingungen macht deutlich, daß die Wassertemperaturen in den Sorrenter Höhlen mit Ausnahme der tiefen Höhle bei Montalto während der Sommermonate die Strobilationstemperatur von S. planulophorus

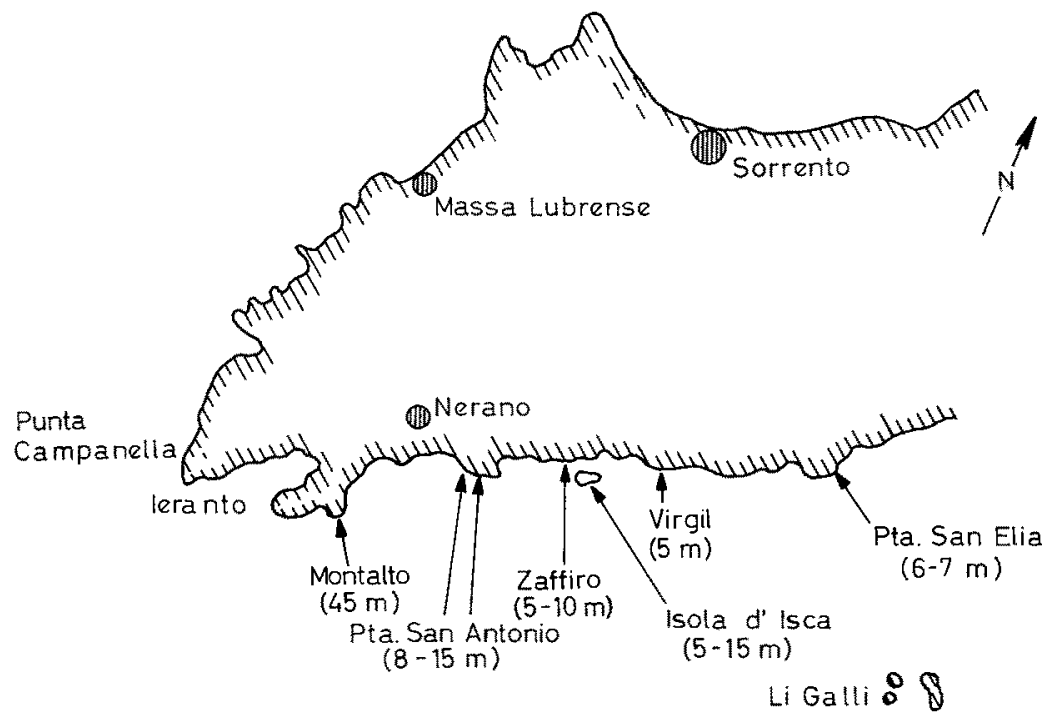

Abb. 5. Lageskizze der untersuchten Unterwasserhöhlen der Halbinsel Sorrent, Golf von Neapel 
erreichen und überschreiten. Hapgood (1959) hat die Temperaturverhältnisse des Golfes von Neapel an mehreren Punkten und in Tiefen von 0 bis $50 \mathrm{~m}$ über einen vollen Jahreszyklus verfolgt. Danach liegen bei der Station D zwischen Sorrent und Capri die Sommertemperaturen von Juni bis Oktober in den Tiefen von 0 bis $10 \mathrm{~m}$ mit Werten von meist $22^{\circ}$ bis $24^{\circ} \mathrm{C}$ ständig über $20^{\circ} \mathrm{C}$ mit einem Minimum von $22,80^{\circ} \mathrm{C}$ und einem Maximum von $26,89^{\circ} \mathrm{C}$. In der Tiefenstufe von $20 \mathrm{~m}$ gelten ähnliche Verhältnisse von August bis Oktober. In der Tiefe von $50 \mathrm{~m}$ aber liegt das Maximum der Temperaturen bei $16,75^{\circ} \mathrm{C}$ im Oktober. Mit dieser Ausnahme wird daher während der Sommermonate in allen Sorrenter Höhlen die für die Vermehrung kritische Strobilationstemperatur erreicht.

Demgegenüber sind die Temperaturverhältnisse des Golfes und der Küste von Marseille auch im Sommer durch niedrigere Temperaturen gekennzeichnet. Nach Bhaud et al. (1967), Thiriot (1966) und Minas (1971) gehören der Golf von Marseille und der westlich angrenzende Golf von Lion zu den kältesten Arealen des Mittelmeeres; ähnliche Temperaturbedingungen werden nur im nördlichen Teil der Adria und im Schwarzen Meer angetroffen. Bei Marseille überschreitet die Temperatur der Oberfläche nur in warmen Sommern und nur kurzfristig eben die $20^{\circ} \mathrm{C}$-Grenze.

In den Horizonten von 5-20 m, in denen die meisten der bei Marseille untersuchten Höhlen liegen (vgl. Werner, 1974, Tab. 1, p. 438), aber bleiben die Wassertemperaturen auch in den warmen Sommermonaten meist unter $20^{\circ} \mathrm{C}$, so daß die für die Strobilation von S. planulophorus notwendigen Temperaturen nicht regelmäßig erreicht werden. Nach allem lassen sich die Unterschiede der Bestandsstärken bei Marseille und Neapel aus den Temperaturverhältnissen hinreichend erklären und begründen.

Ferner ist von allgemeinem Interesse, daß mit $S$. planulophorus nach $S$. eumedusoides eine weitere Höhlenform bekanntgeworden ist. Das ist deswegen von ökologischer und evolutionistischer Bedeutung, weil die beiden Arten die ersten und nach den bisherigen Kenntnissen einzigen echten Höhlenformen unter den Cnidariern des Mittelmeeres darstellen. Nach Riedl (1966) kommen die von ihm in mittelmeerischen Höhlen angetroffenen Cnidaria auch im freien Meer vor.

So liegt es nahe, die abgeänderte Entwicklung als Anpassung an das Höhlenmilieu zu deuten. Die Reduktion der Medusengeneration hat allgemein eine Abkürzung der für meroplanktische Evertebraten stets am meisten gefährdeten vagilen Phase zur Folge, so daß dieses Phänomen als progressiver Evolutionsschritt zu deuten ist. Außerdem verhindert bzw. verringert es die Zerstreuung der Nachkommenschaft, womit die Existenz in Höhlen korreliert ist. Zum gleichen Erfolg führt bei $S$. planulophorus überdies die Abkürzung der planktischen Phase der Planula, die häufig nur 3-4 Tage beträgt, aber bei ungünstigen Bedingungen auf 2-4 Wochen verlängert sein kann. Aussagen über die speziellen Qualitäten des Höhlenmilieus, die für die abgeänderte Entwicklung verantwortlich gemacht werden könnten, sind ohne entsprechende experimentelle Untersuchungen vorerst nicht möglich (vgl. die diesbezügliche Diskussion bei Werner, 1974, p. 459).

Andererseits bedeutet die apogame Entwicklung keinen progressiven, sondern einen regressiven, negativen Evolutionsschritt, da die bisexuelle Vermehrung mit der als Evolutionsfaktor wichtigen Rekombination der Gene verlorengegangen ist. Die Anpassung an das spezielle Milieu der Sackhöhlen hat S. planulophorus offenbar in eine evolutionistische Sackgasse geführt, aus der es keinen Ausweg gibt. 


\section{Diagnose}

Die um die ergänzenden Befunde erweiterte Diagnose lautet jetzt:

Stephanoscyphus planulophorus Werner, 1971: solitärer Scyphopolyp mit schlanker, schwach gebogener Peridermröhre von brauner bis gelblich-weißer Färbung, Oberflächenskulptur mit deutlichen Querringen und Längsstreifen; Formquotient $D$ / $\mathrm{L}=0,07$; Zahnformel $4 \mathrm{P}+4 \mathrm{I}+8 \mathrm{~A}$ (B; 5-7); Ansatz der perradialen Zähne schmal. Weichkörper mit 30-40 Tentakeln mit alternierender Pigmentfärbung an der Basis. Einmündung des entodermalen Ringkanals des Kopfteils in den Gastralraum durch 4 perradiale Poren. Entwicklung mit zunächst typischer polydisker Strobilation; atypische Weiterentwicklung durch direkte Umwandlung der Ephyren in Planulae, die sich nach kurzer planktischer Phase in typischer Weise anheften und zum sessilen Polypen umwandeln. Dadurch im Endergebnis vollständige Reduktion der Medusengeneration, verbunden mit Apogamie. Bicnidom aus holotrichen Isorhizen und heterotrichen mikrobasischen Eurytelen. Herkunft: submarine Höhlen des oberen Fels-Sublitorals bei Marseille (selten) und Neapel (Sorrent, häufig). Wassertiefe $5-30 \mathrm{~m}$.

Danksagungen. Herrn Dr. A. Svoboda, Wien, jetzt Bochum, möchte ich sehr herzlich dafür danken, daß er während eines gemeinsamen Studienaufenthaltes an der Zoologischen Station Neapel im Mai 1975 bei seinen Tauchgängen das Lebendmaterial gesammelt hat, wobei ihm die genaue Kenntnis der Sorrenter Höhlen und seine große Taucherfahrung sehr zustatten kamen. Ebenso danke ich der Leitung und den Mitarbeitern der Zoologischen Station für alle Hilfe während des Studienaufenthaltes. Die Mittel dafür wurden von der Deutschen Forschungsgemeinschaft bereitgestellt, der ich für die langjährige Förderung meiner Arbeiten zu größtem Dank verpflichtet bin.

\section{ZITIERTE LITERATUR}

Bhaud, M., Jacques, G. \& Razouls, C., 1967. Données météorologiques et hydrologiques de la région de Banyuls-Sur-Mer. Année 1965-1966 (Point côtier). - Vie Milieu (B) 18, 137-151.

Chapman, D. M. \& Werner, B., 1972. Structure of a solitary and colonial species of Stephanoscyphus (Scyphozoa, Coronata) with observations on periderm repair. - Helgoländer wiss. Meeresunters. $23,393-421$.

Hapgood, W., 1959. Hydrographic observations in the Bay of Naples (Golfo di Napoli) January 1957-January 1958 (Station Lists). - Pubbl. Staz. zool. Napoli 31, 337-371.

Komai, T., 1935. On Stephanoscyphus and Nausithoë. - Mem. Coll. Sci. Kyoto Univ. (B) 10,289-339.

Komai, T., 1936. On another form of Stephanoscyphus found in waters of Japan. - Mem. Coll. Sci. Kyoto Univ. (B) 11, 175-183.

Komai, T. \& Tokuoka, Y., 1939. Further observations on the strobilation of Stephanoscyphus. - Mem. Coll. Sci. Kyoto Univ. (B) 15, 127-133.

Kramp, P. L., 1959. Stephanoscyphus (Scyphozoa). - Galathea Rep. 1, 173-185.

Kramp, P. L., 1962. Stephanoscyphus corniformis Komai (Scyphozoa). - Lunds Univ. Årsskr. (Avd. 2) $58,1-4$.

Kühn, A., 1910. Die Entwicklung der Geschlechtsindividuen der Hydromedusen. II. Studien zur Ontogenese und Phylogenese der Hydroiden. - Zool. Jo. (Anat. Ontogenie Tiere) 30, 43-174.

Kühn, A., 1913. Entwicklungsgeschichte und Verwandtschaftsbeziehungen der Hydrozoen. I. Die Hydroiden. - Ergebn. Fortschr. Zool. 4, 1-284.

Leloup, E., 1937. Hydropolypes et scyphopolypes recueillis par C. Dawydoff sur les côtes de 1'Tndochine française. - Mém. Mus. r. Hist. nat. Belg. (Sér. 2) 12, 1-73.

Minas, H.-J., 1971. Observations hydrologiques dans le Golf de Marseille (Années 1961 à 1965).Téthys, $3,447-457$.

Naumov, D. V., 1959. [Artliche Verschiedenheiten der Polypengeneration der Coronatae]. (Russ.).Dokl. Akad. Nauk. SSSR 126, 902-904. 
Riedl, R., 1966. Biologie der Meereshöhlen. Parey, Hamburg, 636 pp.

Thiriot, A., 1966. Variations annuelles de la température de l'eau côtiere superficielle de BanyulsSur-Mer. - Vie Milieu 17, 243-252.

Werner, B., 1965. Die Nesselkapseln der Cnidaria, mit besonderer Berücksichtigung der Hydroida. I. Klassifikation und Bedeutung für die Systematik und Evolution. - Helgoländer wiss. Meeresunters. 12, 1-39.

Werner, B., 1966. Stephanoscyphus (Scyphozoa, Coronata) und seine direkte Abstammung von den fossilen Conulata. - Helgoländer wiss. Meeresunters. 13, 317-347.

Werner, B., 1967. Morphologie, Systematik und Lebensgeschichte von Stephanoscyphus (Scyphozoa, Coronata) sowie seine Bedeutung für die Evolution der Scyphozoa. - Zool. Anz. (Suppl.) 30, 297-319.

Werner, B., 1968. Polypengeneration und Entwicklungsgeschichte von Eucheilota maculata (Thecata, Leptomedusae). Mit einem Beitrag zur Methodik der Kultur mariner Hydroiden. - Helgoländer wiss. Meeresunters. 18, 136-168.

Werner, B., 1970. Contribution to the evolution in the genus Stephanoscyphus (Scyphozoa, Coronata) and ecology and regeneration qualities of Stephanoscyphus racemosus. - Publs Seto mar. biol, Lab. 18, 1-20.

Werner, B., 1971. Stephanoscyphus planulophorus n. spec, ein neuer Scyphopolyp mit einem neuen Entwicklungsmodus. - Helgoländer wiss. Meeresunters. 22, 120-140.

Werner, B., 1973. New investigations on systematics and evolution of the class Scyphozoa and the phylum Cnidaria. - Publs Seto mar. biol. Lab. 20, 35-61.

Werner, B., 1974. Stephanoscyphus eumedusoides n. spec,, ein Höhlenpolyp mit einem neuen Entwicklungsmodus. - Helgoländer wiss. Meeresunters. 26, 434-463.

Werner, B., 1979. Coloniality in the Scyphozoa: Cnidaria. In: Biology and systematics of colonial organisms. Ed. by G. Larwood \& B. R. Rosen. Acad. Press, London, 81-103.

Werner, B. \& Hentschel, J., 1983. The apogamous life cycle of Stephanoscyphus planulophorus Werner, 1971 (In prep.). 